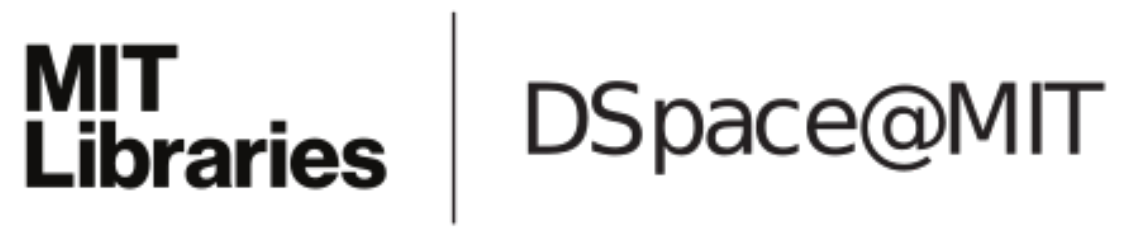

\author{
MIT Open Access Articles
}

Spin-Induced Optical Conductivity in the Spin-Liquid Candidate Herbertsmithite

The MIT Faculty has made this article openly available. Please share how this access benefits you. Your story matters.

Citation: Pilon, D. V. et al. "Spin-Induced Optical Conductivity in the Spin-Liquid Candidate Herbertsmithite." Physical Review Letters 111.12 (2013): n. pag. ( 2013 American Physical Society

As Published: http://dx.doi.org/10.1103/PhysRevLett.111.127401

Publisher: American Physical Society

Persistent URL: http://hdl.handle.net/1721.1/84975

Version: Final published version: final published article, as it appeared in a journal, conference proceedings, or other formally published context

Terms of Use: Article is made available in accordance with the publisher's policy and may be subject to US copyright law. Please refer to the publisher's site for terms of use. 


\title{
Spin-Induced Optical Conductivity in the Spin-Liquid Candidate Herbertsmithite
}

\author{
D. V. Pilon, ${ }^{1}$ C. H. Lui, ${ }^{1}$ T. -H. Han,${ }^{1,4,5}$ D. Shrekenhamer, ${ }^{2}$ A. J. Frenzel,,${ }^{1,3}$ W. J. Padilla, ${ }^{2}$ Y. S. Lee, ${ }^{1}$ and N. Gedik ${ }^{1, *}$ \\ ${ }^{1}$ Massachusetts Institute of Technology, Cambridge, Massachusetts 02139, USA \\ ${ }^{2}$ Department of Physics, Boston College, Chestnut Hill, Massachusetts 02467, USA \\ ${ }^{3}$ Department of Physics, Harvard University, Cambridge, Massachusetts 02138, USA \\ ${ }^{4}$ James Franck Institute and Department of Physics, University of Chicago, Chicago, Illinois 60637, USA \\ ${ }^{5}$ Materials Science Division, Argonne National Laboratory, Argonne, Illinois 60439, USA
}

(Received 15 April 2013; published 18 September 2013)

\begin{abstract}
We report a direct measurement of the low-frequency optical conductivity of large-area single-crystal herbertsmithite, a promising spin-liquid candidate material, by means of terahertz time-domain spectroscopy. In the spectral range below $1.4 \mathrm{THz}$, we observe a contribution to the real part of the in-plane conductivity $\sigma_{a b}(\omega)$ from the spin degree of freedom. This spin-induced conductivity exhibits a powerlaw dependence on frequency $\sigma_{a b}(\omega) \sim \omega^{\beta}$ with $\beta \approx 1.4$. Our observation is consistent with the theoretically predicted low-frequency conductivity arising from an emergent gauge field of a gapless U(1) Dirac spin liquid.
\end{abstract}

DOI: 10.1103/PhysRevLett.111.127401

PACS numbers: 78.20. $-\mathrm{e}, 75.10 . \mathrm{Kt}, 78.30 .-\mathrm{j}$

A quantum spin liquid (QSL) is a state of matter in which antiferromagnetic spins interact strongly, but quantum fluctuations inhibit long-range magnetic order even at zero temperature. The QSL concept was first conceived by Anderson in 1973 [1] and was later suggested to be a possible explanation for high temperature superconductivity in the cuprates [2,3]. The proposed ground state of a QSL, the resonating valence bond (RVB) state, hosts exotic excitations involving spin-charge separation, giving rise to chargeless spin- $1 / 2$ spinons, in contrast to conventional spin-wave excitations (magnons with spin-1) in ordered Mott insulators [4-7]. While QSL's have remained a theoretical construct for decades, recent experiments have provided compelling evidence that the long-sought QSL system is realized in the kagome-lattice antiferromagnet $\mathrm{ZnCu}_{3}(\mathrm{OH})_{6} \mathrm{Cl}_{2}$ (also called herbertsmithite) [8-18] [Fig. 1(a)], as well as in the triangular organic salts $\mathrm{EtMe}_{3} \mathrm{Sb}\left[\mathrm{Pd}(\mathrm{dmit})_{2}\right]_{2}[19,20]$ and $\kappa$-(BEDT-TTF $)_{2} \mathrm{Cu}_{2}(\mathrm{CN})_{3}$ [8,21-23]. In particular, thermodynamic measurements in herbertsmithite exhibit no magnetic order down to $T=50 \mathrm{mK}$ [10], and inelastic neutron scattering measurements exhibit a scattering continuum that is consistent with spinon excitations [16].

Despite this experimental progress in characterizing the QSL state in herbertsmithite, there are still a number of open questions on the nature of the ground state. Specifically, the gauge group of the spin-liquid state and the character of the low-energy spin excitations have yet to be determined. A recent computational study proposes the existence of a $Z_{2}$ spin liquid with a sizable spin gap in herbertsmithite [24]. Thermodynamic and inelastic neutron scattering results, however, show no sign of a spin gap down to $0.1 \mathrm{meV}[10,16]$, suggesting the existence of a gapless U(1) spin liquid state in herbertsmithite. Optical studies have proved difficult due to the chargeless nature of the spinon excitations and the relatively low-energy scale in the spin system. Recent theoretical studies have, however, suggested that spin-charge interactions through an emergent gauge field in a U(1) Dirac spin liquid can give rise to a contribution to the real part of the low-frequency optical conductivity $[25,26]$. In particular, a power-law dependence of the conductivity $(\sigma)$ on photon frequency $(\omega)$, i.e., $\sigma \sim \omega^{\beta}$, with an exponent $\beta=2$, is expected at frequencies far below the charge gap. A power-law dependence in conductivity has previously been reported in another spin-liquid candidate [the organic compound $\kappa$-(BEDT-TTF $)_{2} \mathrm{Cu}_{2}(\mathrm{CN})_{3}$ [27]], but the observed behavior did not agree quantitatively with the theoretical predictions. Direct measurement of such power-law optical conductivity has yet to be performed in herbertsmithite and is therefore a great step toward elucidating the nature of its QSL ground state and the structure of its low-energy excitations.

In this Letter, we report a direct observation of the spininduced low-frequency optical conductivity in herbertsmithite. This experiment is made possible by the recent successful growth of large-area single crystals of herbertsmithite [28]. By using terahertz (THz) time-domain spectroscopy, we have measured the real part of the optical conductivity of herbertsmithite as a function of temperature and magnetic field in the spectral range 0.6-2.2 THz. Remarkably, the in-plane conductivity $\sigma_{a b}(\omega)$, which is associated with the spin-liquid state in the kagome $(a b)$ planes of herbersmithite, is found to depend on frequency as $\sigma_{a b}(\omega) \sim \omega^{\beta}$ with $\beta \approx 1.4$, a result compatible with the theoretical predictions $[25,26]$. The observed powerlaw conductivity also exhibits temperature dependence opposite to that expected for an insulator, and is absent in the out-of-plane direction, as expected for a two-dimensional gapless spin-liquid state. Our findings 


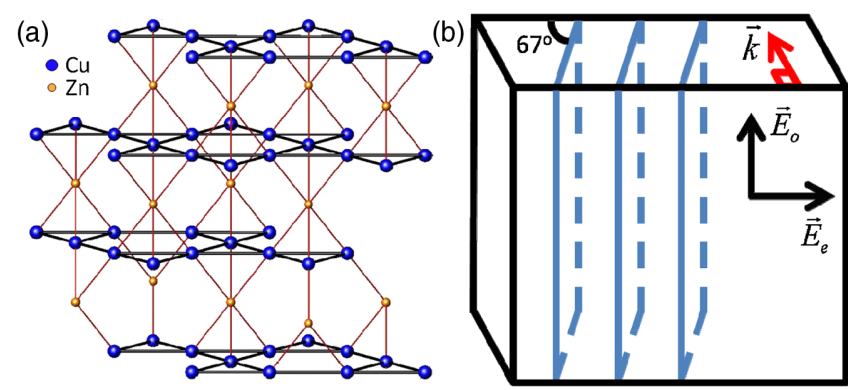

FIG. 1 (color online). (a) Schematic lattice structure of herbertsmithite. The crystal is composed of layers of spin-1/2 copper atoms (blue dots) arranged in the kagome pattern in the $a b$ plane, separated by nonmagnetic zinc atoms (orange dots) along the perpendicular $c$ axis. The chlorine, oxygen, and hydrogen atoms in herbertsmithite are neglected here for simplicity. (b) The orientation of the herbertsmithite sample used in our experiment. The $a b$ planes are aligned vertically, making an angle of $67^{\circ}$ with the sample surface. $E_{o}, E_{e}$, and $k$ denote, respectively, the ordinary and extraordinary optical axes of the crystal and the wave vector of the incident terahertz beam.

are consistent with the predicted low-frequency absorption arising from an emergent gauge field in a gapless $U(1)$ Dirac spin liquid.

In our experiment, we investigated a large singlecrystal sample of herbertsmithite with dimensions $3 \times 6 \times 0.8 \mathrm{~mm}$. The sample was characterized by neutron diffraction, anomalous $\mathrm{x}$-ray diffraction, and thermodynamic measurements, with results compatible with those of powder samples [28]. As shown in Fig. 1(a), herbertsmithite has a layered structure, with spin-1/2 copper atoms in a kagome pattern forming planes separated by nonmagnetic zinc atoms. This material exhibits strong in-plane $(a b)$ antiferromagnetic interactions with Curie-Weiss temperature $\Theta_{\mathrm{CW}}=-300 \mathrm{~K}$, a charge gap of $\sim 2 \mathrm{eV}$, and a spinon gap of less than $0.1 \mathrm{meV}$, as well as an exchange energy $J \approx 17 \mathrm{meV}(200 \mathrm{~K})$, described by a Heisenberg model with negligible out-of-plane $(c)$ interactions [10]. Despite the strong interactions in this material, geometric frustration prevents the formation of any magnetic order down to at least $T=50 \mathrm{mK}$ [10].

We measured the THz in-plane conductivity $\sigma_{a b}(\omega)$ that is associated with the spin liquid state in the herbertsmithite crystal by $\mathrm{THz}$ time-domain spectroscopy. The experiment was performed using an amplified Ti:Sapphire laser system, which generated pulses with 800-nm central wavelength, $100-\mathrm{fs}$ pulse duration and $5-\mathrm{kHz}$ repetition rate. The $\mathrm{THz}$ radiation was generated via optical rectification in a $\langle 110\rangle$-oriented ZnTe crystal, focused onto the sample using off-axis parabolic mirrors, and subsequently detected via free space electro-optic sampling in a second $\mathrm{ZnTe}$ crystal. The $\mathrm{THz}$ radiation was polarized along the kagome $(a b)$ planes using wire-grid polarizers. We recorded the $\mathrm{THz}$ electric field transmitted through the herbertsmithite sample, and, as a reference, the $\mathrm{THz}$ field transmitted through vacuum. From the Fourier transformed frequency-domain fields for the sample and reference, we extracted the frequency-dependent optical conductivity, taking into account the sample geometry. All conductivities reported in this Letter are the real components.

Figure 2(a) displays the in-plane optical conductivity spectra $\sigma_{a b}(\omega)$ in the frequency range $0.6-2.2 \mathrm{THz}$ at temperatures from 4 to $150 \mathrm{~K}$. The conductivity spectra can be described by two components. The higherfrequency component, which is significant for frequencies $>1.4 \mathrm{THz}$, can be attributed to a phonon absorption with a resonance at $\sim 3 \mathrm{THz}$ [29]. Here we focus on the lowerfrequency component, which dominates the absorption at frequencies $<1.4 \mathrm{THz}$. This component can be described by a power law with a small exponent $\sigma_{a b}(\omega) \sim \omega^{\beta}$, where $\beta \approx 1.4$. (As discussed in the Supplemental Material, an exponent $\beta$ between 1 and 2 is still compatible with the data due to the limited frequency range in our measurement [29].) Such an absorption behavior is distinct from that expected in ordered Mott insulators, which typically exhibit $\omega^{4}$ frequency-dependent conductivity at low frequencies arising from the spin-wave excitations [25]. In the following discussion, we will provide evidence that the $\omega^{\beta}$ absorption arises from spinon excitations in herbertsmithite.

First, we observe a noticeable enhancement of the $\omega^{\beta}$ absorption component as the temperature decreases from 150 to $4 \mathrm{~K}$ [Fig. 2(b)]. The increase of absorption at lower temperature is an anomalous phenomenon for insulating materials, where light absorption far below the band gap typically decreases at low temperature due to the freezing of phonons and thermally excited carriers. The observed unusual temperature dependence immediately indicates that the underlying absorption mechanism is of exotic origin. Indeed, the temperature dependence of $\sigma_{a b}(\omega)$ is reminiscent of that of metals, suggesting that $\sigma_{a b}(\omega)$ is associated with a gapless or nearly gapless spin system in herbertsmithite. Our results are consistent with a similar phenomenon in the Raman scattering of herbertsmithite, where a continuum of Raman signal due to spinon excitations is found to increase with decreasing temperature at $T<50 \mathrm{~K}$ [14]. From the lower bound of our measured frequency range, we estimate that the spin gap in herbertsmithite, if it exists, should not be larger than $0.6 \mathrm{THz}(\sim 2 \mathrm{meV})$. This value is consistent with the upper bound of the spin gap $(\sim 0.1 \mathrm{meV})$ estimated by other experimental studies $[10,16]$.

Second, the $\omega^{\beta}$ absorption component disappears for light linearly polarized in the direction perpendicular to the kagome planes. We have measured the out-of-plane conductivity $\sigma_{c}(\omega)$ along the $c$ axis of the herbertsmithite crystal at different temperatures [29]. The $\sigma_{c}(\omega)$ spectra resemble the tail of a phonon resonance at $>3 \mathrm{THz}$ [Fig. 3]. In particular, $\sigma_{c}(\omega)$ at $T=4 \mathrm{~K}$ drops rapidly to 

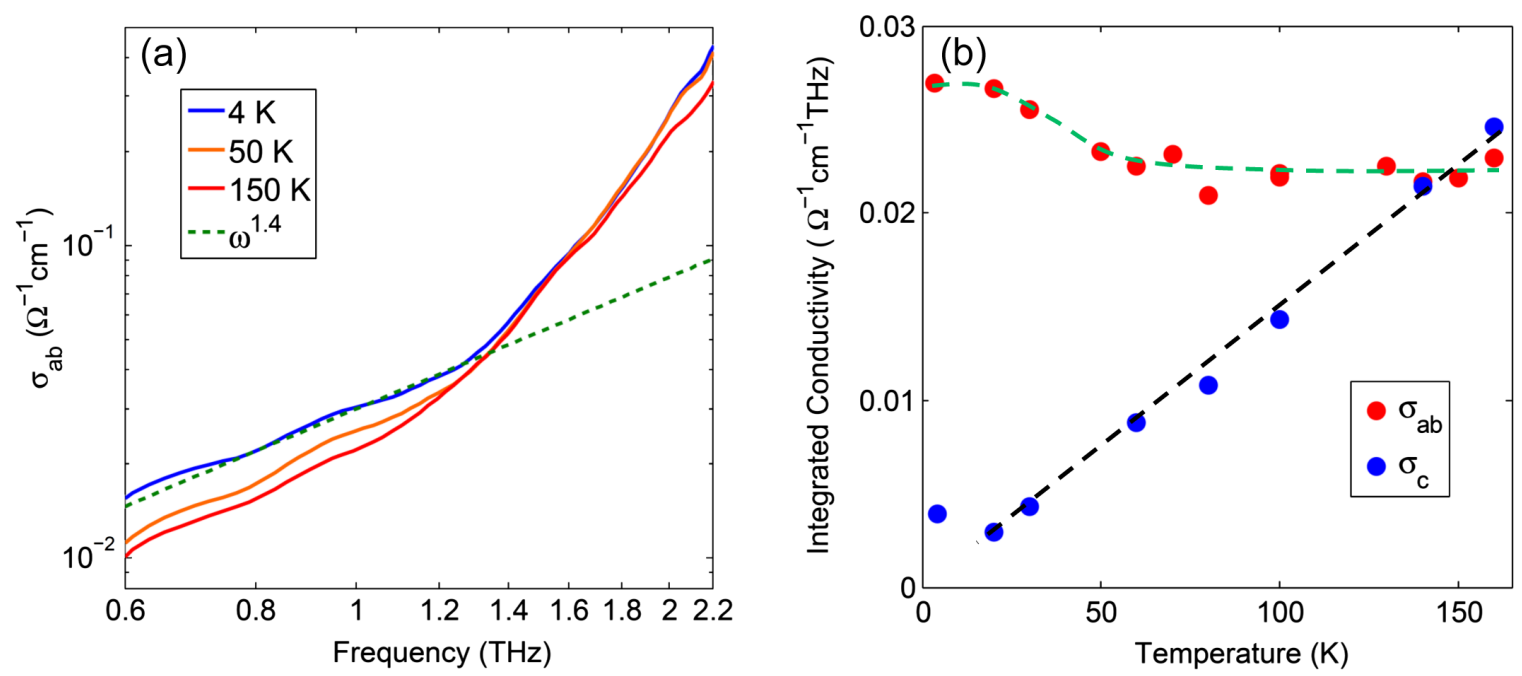

FIG. 2 (color online). (a) The in-plane real optical conductivity $\sigma_{a b}$ in the spectral range $0.6-2.2 \mathrm{THz}$ at temperatures $T=4,50$, and $150 \mathrm{~K}$. The spectra consist of a high-frequency component arising from a phonon resonance at $\sim 3 \mathrm{THz}$, and a low-frequency component that exhibits a power-law dependence on frequency as $\sigma_{a b} \sim \omega^{\beta}$ with $\beta \approx 1.4$ (dotted line). The data are plotted on a log$\log$ scale to highlight this power-law behavior. (b) The integrated values of the in-plane conductivity $\sigma_{a b}$ from panel (a) and of the outof-plane conductivity $\sigma_{c}$ from Fig. 3 from 0.6 to $1.4 \mathrm{THz}$ for temperatures $T=4-160 \mathrm{~K}$. $\sigma_{a b}$ decreases as the temperature increases up to $T \sim 60 \mathrm{~K}$, after which it remains constant. In contrast, $\sigma_{c}$ increases monotonically with temperature. Dashed lines are guides to the eye.

zero at frequencies lower than $1 \mathrm{THz}$, in contrast to the $\omega^{\beta}$ behavior observed in the in-plane conductivity $\sigma_{a b}(\omega)$. In addition, the magnitude of $\sigma_{c}(\omega)$ is reduced as the temperature decreases [Figs. 2(b) and 3], a typical behavior of phonon absorption. The absence of the $\omega^{\beta}$ absorption component in the out-of-plane direction indicates that it is adherent to the in-plane properties of the sample. Due to

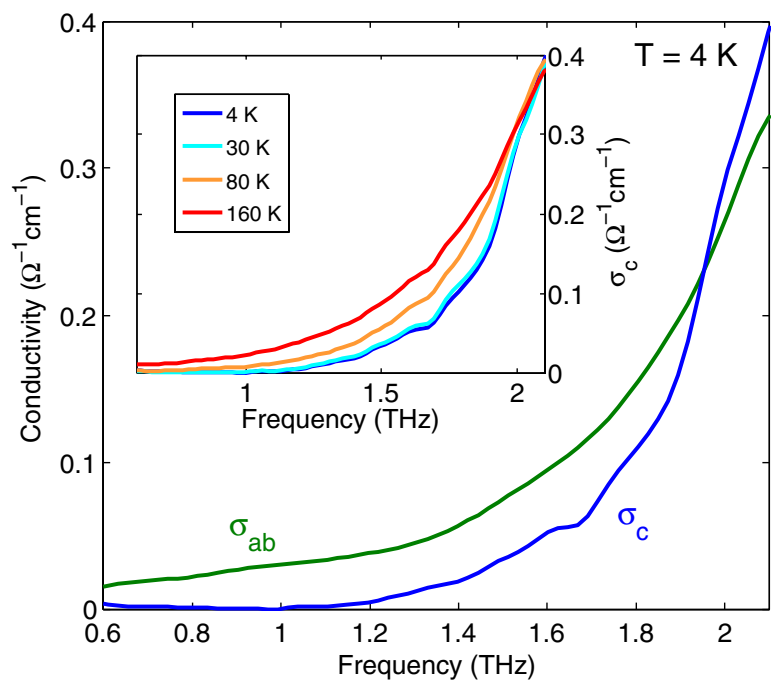

FIG. 3 (color online). Out-of-plane real optical conductivity of herbertsmithite $\left(\sigma_{c}\right)$ (blue line), measured in the spectral range 0.6-2.2 THz at $T=4 \mathrm{~K}$, in comparison with the in-plane conductivity $\sigma_{a b}$ (green line). The inset displays the $\sigma_{c}$ spectra at $T=4,30,80$, and $160 \mathrm{~K}$. The magnitude of $\sigma_{c}$ increases with temperature. the quasi-two-dimensional nature of the spin system in herbertsmithite, where the spin excitations are confined to move only in the kagome planes, our result strongly suggests that the $\omega^{\beta}$ absorption found only within the planes is associated with the spin degree of freedom in herbertsmithite.

Third, the $\omega^{\beta}$ absorption is insensitive to the presence of a strong magnetic field. We have measured the in-plane conductivity $\sigma_{a b}(\omega)$ in the spectral range $0.6-2.2 \mathrm{THz}$ in magnetic fields from 0 to $7 \mathrm{~T}$ at $T=6 \mathrm{~K}$ [Fig. 4] using a superconducting magnet cryostat. We did not find any systematic changes of the absorption spectrum with the magnetic field. This observation rules out magnetic impurities as the source of the observed $\mathrm{THz}$ absorption [29]. Indeed, the result is consistent with the expected behavior of the spinon excitations in a spin liquid, which generally exhibit a field-independent energy spectrum except in extreme conditions (such as in $B>12 \mathrm{~T}$ or $T<0.5 \mathrm{~K}$, where a phase transition may occur) [30,31].

Finally, the observed frequency dependence and magnitude of $\sigma_{a b}$ agree well with those predicted for spininduced absorption in a spin liquid. Recent theory shows that in a gapless $U(1)$ Dirac spin liquid a power-law optical conductivity $\sigma \sim \omega^{\beta}$ with $\beta=2$ can arise from spincharge interactions through an internal gauge field, and the calculated absorption magnitude is compatible with our experimental data $[25,26]$. These theoretical studies point out that observation of power-law conductivity inside the Mott gap stronger than $\omega^{4}$, that is, with an exponent smaller than four, is strong evidence for the importance of gauge fields in herbertsmithite. Similar power-law 


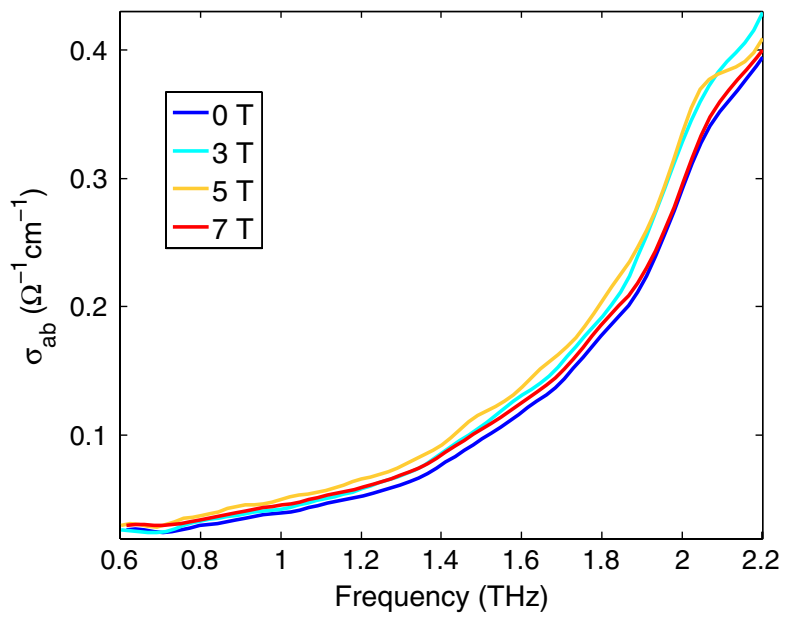

FIG. 4 (color online). In-plane real conductivity of herbertsmithite $\left(\sigma_{a b}\right)$ measured in the spectral range $0.6-2.2 \mathrm{THz}$ at magnetic fields $0,3,5$, and $7 \mathrm{~T}$. No systematic magnetic-field dependence is observed. The small variation between measurements is attributed to experimental uncertainties.

absorption has also been suggested for a gapped $Z_{2}$ spin liquid due to modulation of the Dzyaloshinkii-Moriya (DM) interaction, but the predicted absorption magnitude is 3 orders of magnitude too small to match our data [26]. Our results therefore favor the existence of a U(1) spin liquid state with an emergent gauge field in herbertsmithite.

In conclusion, we have observed a power-law component $\omega^{\beta}$ with $\beta=1 \sim 2$ in the low-frequency in-plane optical conductivity of spin-liquid candidate herbertsmithite. Detailed analysis shows that the absorption arises from spinon excitations. Our results agree with theoretical predictions based on spin-charge coupling through an emergent gauge field in a gapless Dirac spin liquid, and put an upper bound of $2 \mathrm{meV}$ on the size of the spin gap. This discovery hints at the existence of a gauge field in a spin liquid, which is of profound significance to spin-liquid research. More generally, our research demonstrates terahertz time-domain spectroscopy to be an effective probe to study quantum spin liquids. The conductivity measurements have potential to extend to the $\mathrm{GHz}$ and $\mathrm{MHz}$ frequency range via electronic methods, which, combined with sub-Kelvin temperatures, may allow one to probe the extreme limit of low-energy excitations and provide a definitive answer regarding the nature of the ground state in herbertsmithite.

We thank P. A. Lee, A. Potter, T. Senthil, and C. Wang for discussions. This work was supported by the US Department of Energy (DOE), Office of Basic Energy Sciences (BES), Division of Materials Sciences and Engineering: Grants No. DE-FG02-08ER46521 (optical characterization), No. DE-SC0006423 (technique development), and No. DE-FG02-07ER46134 (material synthesis).
*Corresponding author. gedik@mit.edu

[1] W. P. Anderson, Mater. Res. Bull. 8, 153 (1973).

[2] W. P. Anderson, Science 235, 1196 (1987).

[3] P. A. Lee, N. Nagaosa, and X.-G. Wen, Rev. Mod. Phys. 78, 17 (2006).

[4] Y. Ran, M. Hermele, P. A. Lee, and X.-G Wen, Phys. Rev. Lett. 98, 117205 (2007).

[5] S.-S. Lee and P. A. Lee, Phys. Rev. Lett. 95, 036403 (2005).

[6] L. Balents, Nature (London) 464, 199 (2010).

[7] S.-S. Lee, P. A. Lee, and T. Senthil, Phys. Rev. Lett. 98, 067006 (2007).

[8] P. A. Lee, Science 321, 1306 (2008).

[9] M.P. Shores, E. A. Nytko, B.M. Bartlett, and D. G. Nocera, J. Am. Chem. Soc. 127, 13462 (2005).

[10] J.S. Helton et al., Phys. Rev. Lett. 98, 107204 (2007).

[11] P. Mendels, F. Bert, M. A. de Vries, A. Olariu, A. Harrison, F. Duc, J. C. Trombe, J.S. Lord, A. Amato, and C. Baines, Phys. Rev. Lett. 98, 077204 (2007).

[12] T. Imai, E. A. Nytko, B. M. Bartlett, M. P. Shores, and D. G. Nocera, Phys. Rev. Lett. 100, 077203 (2008).

[13] A. Olariu, P. Mendels, F. Bert, F. Duc, J. Trombe, M. de Vries, and A. Harrison, Phys. Rev. Lett. 100, 087202 (2008).

[14] D. Wulferding, P. Lemmens, P. Scheib, J. Röder, P. Mendels, S. Chu, T. Han, and Y.S. Lee, Phys. Rev. B 82, 144412 (2010).

[15] D. E. Freedman, T.H. Han, A. Prodi, P. Muller, Q.-Z. Huang, Y.-S. Chen, S.M. Webb, Y.S. Lee, T.M. McQueen, and D. G. Nocera, J. Am. Chem. Soc. 132, 16185 (2010).

[16] T.-H. Han, J.S. Helton, S. Chu, D. G. Nocera, J. A. Rodriguez-Rivera, C. Broholm, and Y.S. Lee, Nature (London) 492, 406 (2012).

[17] V. R. Shaginyan, A.Z. Msezane, and K. G. Popov, Phys. Rev. B 84, 060401 (2011).

[18] M. A. deVries, K. V. Kamenev, W. A. Kockelmann, J. Sanchez-Benitez, and A. Harrison, Phys. Rev. Lett. 100, 157205 (2008).

[19] T. Itou, A. Oyamada, S. Maegawa, M. Tamura, and R. Kato, Phys. Rev. B 77, 104413 (2008).

[20] M. Yamashita, N. Nakata, Y. Senshu, M. Nagata, H. M. Yamamoto, R. Kato, T. Shibauchi, and Y. Matsuda, Science 328, 1246 (2010).

[21] S. Yamashita, Y. Nakazawa, M. Oguni, Y. Oshima, H. Nojiri, Y. Shimizu, K. Miyagawa, and K. Kanoda, Nat. Phys. 4, 459 (2008).

[22] F. L. Pratt, P. J. Baker, S. J. Blundell, T. Lancaster, S. Ohira-Kawamura, C. Baines, Y. Shimizu, K. Kanoda, I. Watanabe, and G. Saito, Nature (London) 471, 612 (2011).

[23] I. Kézsmárki, Y. Shimizu, G. Mihály, Y. Tokura, K. Kanoda, and G. Saito, Phys. Rev. B 74, 201101(R) (2006).

[24] S. Yan, D. A. Huse, and S. R. White, Science 332, 1173 (2011).

[25] T.-K. Ng and P. A. Lee, Phys. Rev. Lett. 99, 156402 (2007). 
[26] A. C. Potter, T. Senthil, and P. A. Lee, Phys. Rev. B 87, 245106 (2013).

[27] S. Elsasser, D. Wu, M. Dressel, and J. A. Schleuter, Phys. Rev. B 86, 155150 (2012).

[28] T. H. Han, J. S. Helton, S. Chu, A. Prodi, D. K. Singh, C. Mazzoli, P. Müller, D. G. Nocera, and Y. S. Lee, Phys. Rev. B 83, 100402(R) (2011).
[29] See Supplemental Material at http://link.aps.org/ supplemental/10.1103/PhysRevLett.111.127401 for more information.

[30] M. Jeong, F. Bert, P. Mendels, F. Duc, J. C. Trombe, M. A. de Vries, and A. Harrison, Phys. Rev. Lett. 107, 237201 (2011).

[31] Y. Ran, W.H. Ko, P. A. Lee, and X.-G. Wen, Phys. Rev. Lett. 102, 047205 (2009). 